\title{
A NOTE ON STRONG APPROXIMATION OF SDES WITH SMOOTH COEFFICIENTS THAT HAVE AT MOST LINEARLY GROWING DERIVATIVES
}

\author{
THOMAS MÜLLER-GRONBACH AND LARISA YAROSLAVTSEVA
}

\begin{abstract}
Recently, it has been shown in [Jentzen, A., Müller-Gronbach, T., and Yaroslavtseva, L., Commun. Math. Sci., 14, 2016] that there exists a system of autonomous stochastic differential equations (SDE) on the time interval $[0, T]$ with infinitely differentiable and bounded coefficients such that no strong approximation method based on evaluation of the driving Brownian motion at finitely many fixed times in $[0, T]$, e.g. on an equidistant grid, can converge in absolute mean to the solution at the final time with a polynomial rate in terms of the number of Brownian motion values that are used. In the literature on strong approximation of SDEs, polynomial error rate results are typically achieved under the assumption that the first order derivatives of the coefficients of the equation satisfy a polynomial growth condition. This assumption is violated for the pathological SDEs from the above mentioned negative result. However, in the present article we construct an SDE with smooth coefficients that have first order derivatives of at most linear growth such that the solution at the final time can not be approximated with a polynomial rate, whatever method based on observations of the driving Brownian motion at finitely many fixed times is used. Most interestingly, it turns out that using a method that adjusts the number of evaluations of the driving Brownian motion to its actual path, the latter SDE can be approximated with rate 1 in terms of the average number of evaluations that are used. To the best of our knowledge, this is only the second example in the literature of an SDE for which there exist adaptive methods that perform superior to non-adaptive ones with respect to the convergence rate.
\end{abstract}

\section{INTRODUCTION}

Let $d, m \in \mathbb{N}, T \in(0, \infty)$, consider a $d$-dimensional system of autonomous stochastic differential equations (SDE)

$$
\begin{aligned}
d X(t) & =\mu(X(t)) d t+\sigma(X(t)) d W(t), \quad t \in[0, T], \\
X(0) & =x_{0}
\end{aligned}
$$

with a deterministic initial value $x_{0} \in \mathbb{R}^{d}$, a drift coefficient $\mu: \mathbb{R}^{d} \rightarrow \mathbb{R}^{d}$, a diffusion coefficient $\sigma: \mathbb{R}^{d} \rightarrow \mathbb{R}^{d \times m}$ and an $m$-dimensional driving Brownian motion $W$, and assume that (1) has a unique strong solution $(X(t))_{t \in[0, T]}$. A fundamental problem in the numerical analysis of SDEs is to characterize when the solution at the final time $X(T)$ can be approximated with a polynomial error rate based on finitely many evaluations of the driving Brownian motion $W$ in terms of explicit regularity conditions on the coefficients $\mu$ and $\sigma$.

It is well-known that if the coefficients $\mu$ and $\sigma$ are globally Lipschitz continuous then the classical Euler-Maruyama scheme achieves the rate of convergence 1/2, see [26]. Moreover, the recent literature on numerical approximation of SDEs contains a number of results 
on approximation schemes that are specifically designed for SDEs with non-Lipschitz coefficients and achieve polynomial convergence rates under weaker conditions on $\mu$ and $\sigma$, see e.g. [16, 13, 18, 25, 35, 33, 34, 3, 21, 4, 6] for SDEs with globally monotone coefficients and e.g. [2, 9, 5, 1, 30, 17, 19, 23, 24, 31, 12, for SDEs with possibly non-monotone coefficients.

On the other hand, it has recently been shown in [20] that for any sequence $\left(a_{n}\right)_{n \in \mathbb{N}} \subset(0, \infty)$, which may converge to zero arbitrarily slowly, there exists an SDE (1) with $d=4$ and $m=1$ and with infinitely differentiable and bounded coefficients $\mu$ and $\sigma$ such that no sequence of approximations $\widehat{X}_{n}(T)$ of $X(T)$, where $\widehat{X}_{n}(T)$ is based on $n$ evaluations of the driving Brownian motion $W$ at fixed time points in $[0, T]$, can converge to $X(T)$ in absolute mean faster than the given sequence $\left(a_{n}\right)_{n \in \mathbb{N}}$. More formally, for this SDE one has for every $n \in \mathbb{N}$,

$$
\inf _{\substack{s_{1}, \ldots, s_{n} \in[0, T] \\ u: \mathbb{R}^{n} \rightarrow \mathbb{R}^{4} \text { measurable }}} \mathbb{E}\left[\left|X(T)-u\left(W\left(s_{1}\right), \ldots, W\left(s_{n}\right)\right)\right|\right] \geq a_{n} .
$$

In [8] it has been proven that the negative result (2) can even be achieved with $m=1$ and $d=2$ in place of $d=4$. In particular, (2) implies that there exists an SDE (11) with infinitely differentiable and bounded coefficients $\mu$ and $\sigma$ such that its solution at the final time can not be approximated with a polynomial mean error rate based on evaluations of the driving Brownian motion $W$ at finitely many fixed time points in $[0, T]$, i.e., for every $\alpha>0$,

$$
\lim _{n \rightarrow \infty}\left(n^{\alpha} \cdot \inf _{\substack{s_{1}, \ldots, s_{n} \in[0, T] \\ u: \mathbb{R}^{n m} \rightarrow \mathbb{R}^{d}}} \mathbb{E}\left[\left|X(T)-u\left(W\left(s_{1}\right), \ldots, W\left(s_{n}\right)\right)\right|\right]\right)=\infty .
$$

We add that the latter statement for the special case when the approximation $u\left(W\left(s_{1}\right), \ldots, W\left(s_{n}\right)\right)$ is given by the Euler-Maruyama scheme with time step $1 / n$ has first been shown in [10].

The proof of the negative result (2) in [20] is constructive. Each of the respective SDEs is given by $X(0)=0$ and

$$
\begin{aligned}
& d X_{1}(t)=d t, \quad d X_{2}(t)=f\left(X_{1}(t)\right) d W(t), \quad d X_{3}(t)=g\left(X_{1}(t)\right) d W(t), \\
& d X_{4}(t)=h\left(X_{1}(t)\right) \cdot \cos \left(X_{2}(t) \cdot \psi\left(X_{3}(t)\right)\right) d t
\end{aligned}
$$

for $t \in[0, T]$, where $f, g, h: \mathbb{R} \rightarrow \mathbb{R}$ are infinitely differentiable, bounded, nonzero and satisfy $\{f \neq 0\} \subset\left(-\infty, \tau_{1}\right],\{g \neq 0\} \subset\left[\tau_{1}, \tau_{2}\right],\{h \neq 0\} \subset\left[\tau_{2}, T\right], \int_{\tau_{2}}^{T} h(t) d t \neq 0$ and $\inf _{x \in\left[0, \tau_{1} / 2\right]}\left|f^{\prime}(x)\right|>$ 0 for some $0<\tau_{1}<\tau_{2}<T$, and $\psi: \mathbb{R} \rightarrow(0, \infty)$ is infinitely differentiable, strictly increasing and satisfies $\lim _{x \rightarrow \infty} \psi(x)=\infty$. Under these assumptions the fourth component of the solution of the SDE (4) at the final time is given by

$$
X_{4}(T)=\cos \left(\int_{0}^{\tau_{1}} f(t) d W(t) \cdot \psi\left(\int_{\tau_{1}}^{\tau_{2}} g(t) d W(t)\right)\right) \cdot \int_{\tau_{2}}^{T} h(t) d t
$$

and there exist $c_{1}, c_{2}, c_{3} \in(0, \infty)$ such that for every $n \in \mathbb{N}$,

$$
\inf _{\substack{s_{1}, \ldots, s_{n} \in[0, T] \\ u: \mathbb{R}^{n} \rightarrow \mathbb{R} \text { measurable }}} \mathbb{E}\left[\left|X_{4}(T)-u\left(W\left(s_{1}\right), \ldots, W\left(s_{n}\right)\right)\right|\right] \geq c_{1} \cdot \exp \left(-c_{2} \cdot\left(\psi^{-1}\left(c_{3} \cdot n^{3 / 2}\right)^{2}\right),\right.
$$

see Corollary 4.1 in [20].

It follows from (6) that if

$$
\forall q \in(0, \infty): \quad \lim _{x \rightarrow \infty} \exp \left(-q x^{2}\right) \cdot \psi(x)=\infty
$$


then a polynomial rate of convergence to zero of the left hand side in (6) can not be achieved, see Corollary 4.2 in [20]. On the other hand it is straightforward to check that the equidistant EulerMaruyama scheme for the SDE (44) achieves a polynomial mean error rate if the derivative $\psi^{\prime}$ of $\psi$ is of at most polynomial growth. The latter two facts are reflected in the growth properties of the first order derivatives of the coefficients $\mu$ and $\sigma$ of the SDE (44). All of the first order derivatives of $\mu$ and $\sigma$ are globally bounded, up to the derivatives

$$
\frac{\partial \mu_{4}}{\partial x_{2}}(x)=-h\left(x_{1}\right) \cdot \psi\left(x_{3}\right) \cdot \sin \left(x_{2} \cdot \psi\left(x_{3}\right)\right), \quad \frac{\partial \mu_{4}}{\partial x_{3}}(x)=-h\left(x_{1}\right) \cdot x_{2} \cdot \psi^{\prime}\left(x_{3}\right) \cdot \sin \left(x_{2} \cdot \psi\left(x_{3}\right)\right),
$$

which are both of at most polynomial growth if and only if $\psi^{\prime}$ is of at most polynomial growth.

For the vast majority of SDEs with locally Lipschitz continuous coefficients used for modelling in applications it holds that the first order derivatives of the coefficients are of at most polynomial growth. Moreover, a polynomial growth condition on the first order derivatives of the coefficients of an SDE is one of the standing assumptions in the literature when polynomial mean error rates are obtained under monotonicity conditions, see e.g. [16, 13, 18, 35, 33, 34, 3, 21, 4, 6]. Therefore it is important to investigate whether a sub-polynomial rate of convergence as in (3) may also happen when the first order derivatives of the coefficients are of at most polynomial growth.

This question can easily be answered with a yes. For the choice $\psi(x)=\exp \left(x^{3}\right)$, which satisfies (7), the random variable $X_{4}(T)$ in (5) can also be obtained as the fifth component of the solution at the final time of an SDE given by $Y(0)=(0,0,0,1,0)$ and

$$
\begin{aligned}
& d Y_{1}(t)=d t, \quad d Y_{2}(t)=f\left(Y_{1}(t)\right) d W(t), \quad d Y_{3}(t)=g\left(Y_{1}(t)\right) d W(t), \\
& d Y_{4}(t)=u\left(Y_{1}(t)\right) \cdot Y_{3}^{3}(t) \cdot Y_{4}(t) d t, \quad d Y_{5}(t)=v\left(Y_{1}(t)\right) \cdot \cos \left(Y_{2}(t) \cdot Y_{4}(t)\right) d t
\end{aligned}
$$

for $t \in[0, T]$, where $f, g, h$ satisfy the conditions stated below the SDE (44) and, additionally, $f^{\prime}$ is bounded, and $u, v: \mathbb{R} \rightarrow \mathbb{R}$ are infinitely differentiable and satisfy $\{u \neq 0\} \subset\left[\tau_{2}, \tau_{3}\right]$, $\{v \neq 0\} \subset\left[\tau_{3}, T\right], \int_{\tau_{2}}^{\tau_{3}} u(s) d s=1$ and $\int_{\tau_{3}}^{T} v(s) d s=\int_{\tau_{2}}^{T} h(t) d t$ for some $\tau_{3} \in\left(\tau_{2}, T\right)$. Clearly, the coefficients of the SDE (8) have first order derivatives of at most polynomial growth and $Y_{5}(T)=X_{4}(T)$.

Note, however, that in contrast to the solution $X$ of the SDE (4), the solution $Y$ of the SDE (8) is not integrable at any time $t \in\left[\tau_{3}, T\right]$. In fact, it is easy to see that

$$
\mathbb{E}\left[\sup _{t \in[0, T]}|X(t)|\right]<\infty, \quad \inf _{t \in\left[\tau_{3}, T\right]} \mathbb{E}\left[\left|Y_{4}(t)\right|\right]=\infty
$$

It therefore seems reasonable to modify the question posed above and to ask whether a subpolynomial rate of convergence as in (3) may also happen for an SDE (11) that has smooth coefficients with first order derivatives of at most polynomial growth and a solution $X$ with

$$
\mathbb{E}\left[\sup _{t \in[0, T]}|X(t)|\right]<\infty
$$


In the actual paper we show that the answer to this question is positive as well. More precisely, consider the 7-dimensional SDE given by $X(0)=(0,0,0,0,1,0,0)$ and

$$
\begin{aligned}
& d X_{1}(t)=d t, \quad d X_{2}(t)=f\left(X_{1}(t)\right) d W(t), \\
& d X_{3}(t)=f^{2}\left(X_{1}(t)\right) d t+2 X_{2}(t) \cdot f\left(X_{1}(t)\right) d W(t), \\
& d X_{4}(t)=\frac{1}{4} g^{\prime}\left(X_{1}(t)\right) \cdot X_{3}(t) d t, \quad d X_{5}(t)=X_{4}(t) \cdot X_{5}(t) d t, \\
& d X_{6}(t)=\frac{h^{\prime}\left(X_{1}(t)\right) \cdot X_{5}(t)}{\left(1+X_{2}^{2}(t)\right)^{\frac{1}{2}} \cdot \ln ^{2}\left(2+X_{2}^{2}(t)\right)}, \quad d X_{7}(t)=X_{5}(t) \cdot X_{6}(t) d t
\end{aligned}
$$

for $t \in[0, T]$, where $f, g, h: \mathbb{R} \rightarrow \mathbb{R}$ satisfy the conditions stated below the SDE (4) and, additionally, $g, h \geq 0, f^{\prime}$ is bounded and $\int_{0}^{\tau_{1}} f^{2}(t) d t=\int_{\tau_{1}}^{\tau_{2}} g(t) d t=\int_{\tau_{2}}^{T} h(t) d t=1$. See Example 1 for a possible choice of $f, g, h$. The assumptions on the functions $f, g$ and $h$ imply that all of the first order derivatives of the coefficients of the SDE (10) are of at most linear growth and the solution $X$ of the SDE (10) satisfies the moment condition (9), see Lemmas 1 and 2. Moreover, as a consequence of Theorem 1 we obtain that there exists $c \in(0, \infty)$ such that for all $n \in \mathbb{N}$,

$$
\inf _{\substack{s_{1}, \ldots, s_{n} \in[0, T] \\ \mathbb{R}^{n} \rightarrow \mathbb{R}^{7} \text { measurable }}} \mathbb{E}\left[\left|X(T)-u\left(W\left(s_{1}\right), \ldots, W\left(s_{n}\right)\right)\right|\right] \geq c \cdot \frac{1}{\ln ^{2}(n+1)},
$$

and therefore $X(T)$ can not be approximated with a polynomial mean error rate based on evaluations of the driving Brownian motion $W$ at finitely many fixed time points in $[0, T]$. To the best of our knowledge this is the first result in the literature, which shows that a sub-polynomial rate of convergence may happen even then when the first order derivatives of the coefficients are of at most polynomial growth. It implies in particular that for such SDEs even tamed or projected versions of the Euler-Maruyama scheme or the Milstein scheme, which are specifically designed to cope with the case of superlinearly growing coefficients, see e.g. [18, 33, 3, 21, 44 may fail to achieve a polynomial convergence rate.

The negative result (11) covers only approximations that are based on $n$ evaluations of the driving Brownian motion $W$ at fixed time points $s_{1}, \ldots, s_{n} \in[0, T]$ and leaves it open whether a polynomial mean error rate can be achieved by employing approximations that may adapt the number as well as the location of the evaluation sites of $W$ to the actual path of $W$, e.g. by numerical schemes that adjust the actual step size according to a criterion that is based on the values of $W$ observed so far, see e.g. [7, 28, 29, 27, 32, 22, 14, 15] and the references therein for methods of this type. However, it is well-known that for a huge class of SDEs (11) with globally Lipschitz continuous coefficients $\mu$ and $\sigma$ adaptive approximations of the latter type can not achieve a better rate of convergence compared to what is best possible for non-adaptive ones, which at the same time coincides with the best possible rate of convergence that can be achieved by approximations based on evaluating $W$ at $n$ equidistant times, see [28, 29] and the discussion on asymptotic constants therein. Moreover, it has recently been shown in [36] that the SDE (4) with $\psi$ satisfying (77) can not be approximated with a polynomial mean error rate even then when adaptive approximations may be used.

Up to now there seems to be only one example of an SDE known in the literature, for which adaptive approximations are superior to non-adaptive ones with respect to the convergence rate. In [11] it has been shown that for the one-dimensional squared Bessel process, i.e. the solution of 
the SDE (11) with $d=m=1, \mu=1$ and $\sigma(x)=2 \sqrt{|x|}$, any non-adaptive approximation of $X(T)$ based on $n$ equidistant evaluations of $W$ can only achieve a mean error rate of order $1 / 2$ in terms of $n$, while for every $\alpha \in(0, \infty)$ there exist $c \in(0, \infty)$ and a sequence of approximations $\widehat{X}_{n}(T)$, each based on $n$ sequentially chosen evaluations of $W$, such that $\mathbb{E}\left[\left|X(T)-\widehat{X}_{n}(T)\right|\right] \leq c \cdot n^{-\alpha}$.

Interestingly it turns out that the SDE (10) provides the second example after [11] of an SDE in the literature, for which there exist adaptive approximations that perform superior to non-adaptive ones with respect to the convergence rate. Indeed, there exists $c \in(0, \infty)$ and a sequence of approximations $\widehat{X}_{n}(T)$, each based on $n$ sequentially chosen evaluations of $W$ on average, such that for all $n \in \mathbb{N}$,

$$
\mathbb{E}\left[\left|X(T)-\widehat{X}_{n}(T)\right|\right] \leq c \cdot n^{-1},
$$

see Theorem (2).

We briefly describe the content of the paper. In Section 2 we introduce the particular SDE with smooth coefficients that is studied in the present paper and we discuss moment properties of its solution. Our main results on a sub-polynomial lower error bound for non-adaptive methods (Theorem 1) and a polynomial upper error bound for a suitable adaptive method (Theorem 2) are stated in Section 3. The respective proofs are carried out in Sections 4 and 5. Section 6 is devoted to a discussion of our results and naturally arising open questions.

\section{An SDE With SMooth COEFficients that haVe at MOST Linearly GRowing DERIVATIVES}

Throughout this article we fix the following setting.

Let $T \in(0, \infty)$, let $(\Omega, \mathcal{F}, \mathbb{P})$ be a probability space with a normal filtration $\left(\mathcal{F}_{t}\right)_{t \in[0, T]}$ and let $W:[0, T] \times \Omega \rightarrow \mathbb{R}$ be a standard $\left(\mathcal{F}_{t}\right)_{t \in[0, T]}$-Brownian motion on $(\Omega, \mathcal{F}, \mathbb{P})$.

Let $0<\tau_{1}<\tau_{2}<T$ and let $f, h, g \in C^{\infty}(\mathbb{R}, \mathbb{R})$ satisfy

$$
\{f \neq 0\} \subseteq\left(-\infty, \tau_{1}\right], \quad\{g \neq 0\} \subseteq\left[\tau_{1}, \tau_{2}\right], \quad\{h \neq 0\} \subseteq\left[\tau_{2}, T\right]
$$

as well as

$$
\sup _{t \in\left(-\infty, \tau_{1}\right]}|f(t)|<\infty, \quad \sup _{t \in\left(-\infty, \tau_{1}\right]}\left|f^{\prime}(t)\right|<\infty, \quad \inf _{t \in\left[0, \tau_{1} / 2\right]}\left|f^{\prime}(t)\right|>0, \quad g \geq 0, \quad h \geq 0
$$

and

$$
\int_{0}^{\tau_{1}} f^{2}(t) d t=\int_{\tau_{1}}^{\tau_{2}} g(t) d t=\int_{\tau_{2}}^{T} h(t) d t=1
$$

See the following example for a possible choice of $f, g$ and $h$.

Example 1. Define $\tilde{f}, \widetilde{g}, \widetilde{h}: \mathbb{R} \rightarrow \mathbb{R}$ by

$$
\begin{aligned}
& \widetilde{f}(x)=\mathbb{1}_{\left(-\infty, \tau_{1}\right)}(x) \cdot \exp \left(\frac{1}{x-\tau_{1}}\right), \\
& \widetilde{g}(x)=\mathbb{1}_{\left(\tau_{1}, \tau_{2}\right)}(x) \cdot \exp \left(\frac{1}{\tau_{1}-x}+\frac{1}{x-\tau_{2}}\right), \\
& \widetilde{h}(x)=\mathbb{1}_{\left(\tau_{2}, T\right)}(x) \cdot \exp \left(\frac{1}{\tau_{2}-x}+\frac{1}{x-T}\right) .
\end{aligned}
$$


Then the functions

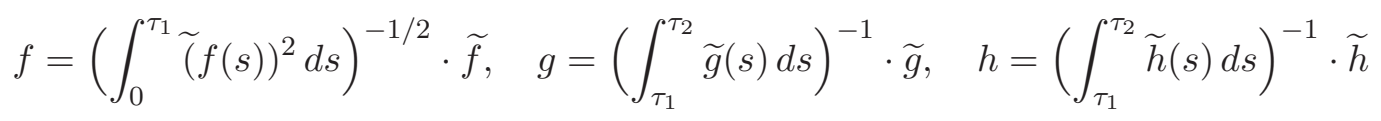

satisfy $f, g, h \in C^{\infty}(\mathbb{R}, \mathbb{R})$ as well as the conditions (12)-(14).

Let $p \in[1, \infty)$ and define $\mu, \sigma: \mathbb{R}^{7} \rightarrow \mathbb{R}^{7}$ as well as $x_{0} \in \mathbb{R}^{7}$ by

$$
\begin{aligned}
\mu(x) & =\left(1,0, f^{2}\left(x_{1}\right), \frac{g^{\prime}\left(x_{1}\right)}{4 p} \cdot x_{3}, x_{4} \cdot x_{5}, \frac{h^{\prime}\left(x_{1}\right) \cdot x_{5}}{\left(1+x_{2}^{2}\right)^{\frac{1}{2 p}} \cdot \ln ^{\frac{2}{p}}\left(2+x_{2}^{2}\right)}, x_{5} \cdot x_{6}\right), \\
\sigma(x) & =\left(0, f\left(x_{1}\right), 2 x_{2} \cdot f\left(x_{1}\right), 0,0,0,0\right) \\
x_{0} & =(0,0,0,0,1,0,0) .
\end{aligned}
$$

Lemma 1. We have $\mu, \sigma \in C^{\infty}\left(\mathbb{R}^{7}, \mathbb{R}^{7}\right)$. Moreover, there exists $c \in(0, \infty)$ such that for all $x \in \mathbb{R}^{7}$,

$$
\sum_{i, j=1}^{7}\left(\left|\frac{\partial \mu_{i}}{\partial x_{j}}(x)\right|+\left|\frac{\partial \sigma_{i}}{\partial x_{j}}(x)\right|\right) \leq c \cdot(1+|x|) .
$$

Proof. Infinite differentiability of $\mu$ and $\sigma$ is an immediate consequence of the definition of these functions and the fact that $f, g, h \in C^{\infty}(\mathbb{R}, \mathbb{R})$. Moreover, it is straightforward to check that there exists $c \in(0, \infty)$ such that for all $i, j \in\{1, \ldots, 7\}$ and $x \in \mathbb{R}^{7}$,

$$
\left|\frac{\partial \sigma_{i}}{\partial x_{j}}(x)\right| \leq c \cdot\left(\left|f\left(x_{1}\right)\right|+\left|f^{\prime}\left(x_{1}\right)\right|\right) \cdot(1+|x|)
$$

and

$$
\left|\frac{\partial \mu_{i}}{\partial x_{j}}(x)\right| \leq c \cdot\left(\left|f\left(x_{1}\right)\right| \cdot\left|f^{\prime}\left(x_{1}\right)\right|+\left|g^{\prime}\left(x_{1}\right)\right|+\left|g^{\prime \prime}\left(x_{1}\right)\right|+\left|h^{\prime}\left(x_{1}\right)\right|+\left|h^{\prime \prime}\left(x_{1}\right)\right|+1\right) \cdot(1+|x|),
$$

which jointly with the fact that $g, h \in C^{\infty}(\mathbb{R}, \mathbb{R})$ and the properties (12) and (13) yields at most linear growth for all first order derivatives of $\mu$ and $\sigma$.

We study the SDE (10) with $m=1, d=7$ and $x_{0}, \mu, \sigma$ given by (16), i.e. $X(0)=(0,0,0,0,1,0,0)^{\top}$ and

$$
\begin{aligned}
& d X_{1}(t)=d t, \quad d X_{2}(t)=f\left(X_{1}(t)\right) d W(t) \\
& d X_{3}(t)=f^{2}\left(X_{1}(t)\right) d t+2 X_{2}(t) \cdot f\left(X_{1}(t)\right) d W(t) \\
& d X_{4}(t)=\frac{1}{4 p} g^{\prime}\left(X_{1}(t)\right) \cdot X_{3}(t) d t, \quad d X_{5}(t)=X_{4}(t) \cdot X_{5}(t) d t \\
& d X_{6}(t)=\frac{h^{\prime}\left(X_{1}(t)\right) \cdot X_{5}(t)}{\left(1+X_{2}^{2}(t)\right)^{\frac{1}{2 p} \cdot \ln ^{\frac{2}{p}}\left(2+X_{2}^{2}(t)\right)}, \quad d X_{7}(t)=X_{5}(t) \cdot X_{6}(t) d t}
\end{aligned}
$$


Observing (12) and using Itô's formula for the component $X_{3}$ it is straightforward to see that the equation (17) has a unique strong solution given by

$$
\begin{aligned}
& X_{1}(t)=t, \quad X_{2}(t)=\int_{0}^{\min \left(t, \tau_{1}\right)} f(s) d W(s), \quad X_{3}(t)=X_{2}^{2}(t), \\
& X_{4}(t)=\frac{1}{4 p} X_{2}^{2}\left(\tau_{1}\right) \cdot g(t), \quad X_{5}(t)=\exp \left(\frac{1}{4 p} X_{2}^{2}\left(\tau_{1}\right) \cdot \int_{0}^{\min \left(t, \tau_{2}\right)} g(s) d s\right), \\
& X_{6}(t)=\frac{X_{5}\left(\tau_{2}\right)}{\left(1+X_{2}^{2}\left(\tau_{1}\right)\right)^{\frac{1}{2 p}} \cdot \ln ^{\frac{2}{p}}\left(2+X_{2}^{2}\left(\tau_{1}\right)\right)} \cdot h(t), \\
& X_{7}(t)=\frac{X_{5}^{2}\left(\tau_{2}\right)}{\left(1+X_{2}^{2}\left(\tau_{1}\right)\right)^{\frac{1}{2 p}} \cdot \ln ^{\frac{2}{p}}\left(2+X_{2}^{2}\left(\tau_{1}\right)\right)} \cdot \int_{0}^{t} h(s) d s
\end{aligned}
$$

for all $t \in[0, T]$. In particular, by (14),

$$
\begin{aligned}
& X_{1}(T)=T, \quad X_{2}(T)=X_{2}\left(\tau_{1}\right)=\int_{0}^{\tau_{1}} f(s) d W(s), \quad X_{3}(T)=X_{2}^{2}(T), \\
& X_{4}(T)=0, \quad X_{5}(T)=\exp \left(\frac{1}{4 p} X_{2}^{2}\left(\tau_{1}\right)\right), \quad X_{6}(T)=0, \\
& X_{7}(T)=\frac{\exp \left(\frac{1}{2 p} X_{2}^{2}\left(\tau_{1}\right)\right)}{\left(1+X_{2}^{2}\left(\tau_{1}\right)\right)^{\frac{1}{2 p}} \cdot \ln ^{\frac{2}{p}}\left(2+X_{2}^{2}\left(\tau_{1}\right)\right)} .
\end{aligned}
$$

Next we discuss integrability properties of the solution $X$.

Lemma 2. We have $X_{2}\left(\tau_{1}\right) \sim \mathcal{N}(0,1)$. Moreover, for all $q \in(0, \infty)$,

$$
\mathbb{E}\left[\sup _{t \in[0, T]}|X(t)|^{q}\right]<\infty \quad \Leftrightarrow \quad q \leq p
$$

Proof. The first statement follows immediately from the definition of $X_{2}\left(\tau_{1}\right)$ and the fact that $\mathbb{E}\left[X_{2}^{2}\left(\tau_{1}\right)\right]=\int_{0}^{\tau_{1}} f^{2}(t) d t=1$, due to (14). Moreover, applying the Burkholder-Davis-Gundy inequality we obtain that for all $q \in(0, \infty)$ there exists $c \in(0, \infty)$ such that

$$
\mathbb{E}\left[\sup _{t \in[0, T]}\left|X_{2}(t)\right|^{q}\right] \leq c \cdot\left(\int_{0}^{T} f^{2}(t) d t\right)^{\frac{q}{2}}=c .
$$

Employing (18), (20) and the properties of $g$ we conclude that for all $q \in(0, \infty)$,

$$
\mathbb{E}\left[\sup _{t \in[0, T]}\left|X_{4}(t)\right|^{q}\right]=\frac{1}{(4 p)^{q}} \cdot \mathbb{E}\left[\left|X_{2}\left(\tau_{1}\right)\right|^{2 q}\right] \cdot \sup _{t \in\left[\tau_{1}, \tau_{2}\right]}(g(t))^{q}<\infty .
$$

Furthermore, (13), (14), (18) and the fact that $X_{2}\left(\tau_{1}\right) \sim \mathcal{N}(0,1)$ imply that for all $q \in(0,2 p)$,

$$
\mathbb{E}\left[\sup _{t \in[0, T]}\left|X_{5}(t)\right|^{q}\right]=\mathbb{E}\left[\exp \left(\frac{q}{4 p} X_{2}^{2}\left(\tau_{1}\right)\right)\right]=\sqrt{\frac{2 p}{2 p-q}} .
$$

By (18), the latter equality and the properties of $h$ we get that for all $q \in(0,2 p)$,

$$
\mathbb{E}\left[\sup _{t \in[0, T]}\left|X_{6}(t)\right|^{q}\right] \leq \frac{1}{\ln ^{\frac{2 q}{p}}(2)} \cdot \mathbb{E}\left[\left|X_{5}\left(\tau_{2}\right)\right|^{q}\right] \cdot \sup _{t \in\left[\tau_{2}, T\right]} h^{q}(t)<\infty .
$$


Finally, by (18) we see that for all $q \in(0, \infty)$,

$$
\mathbb{E}\left[\sup _{t \in[0, T]}\left|X_{7}(t)\right|^{q}\right]=\mathbb{E}\left[\frac{\exp \left(\frac{q}{2 p} X_{2}^{2}\left(\tau_{1}\right)\right)}{\left(1+X_{2}^{2}\left(\tau_{1}\right)\right)^{\frac{q}{2 p}} \cdot \ln \frac{2 q}{p}\left(2+X_{2}^{2}\left(\tau_{1}\right)\right)}\right]=\sqrt{\frac{2}{\pi}} \int_{0}^{\infty} \frac{\exp \left(\frac{q-p}{2 p} \cdot x^{2}\right)}{\left(1+x^{2}\right)^{\frac{q}{2 p}} \cdot \ln \frac{2 q}{p}\left(2+x^{2}\right)} d x,
$$

and the latter quantity is finite if and only if $q \leq p$.

\section{LOWER AND UPPER ERROR BOUNDS}

We study strong approximation of the solution $X$ of the equation (17) at the final time $T$. The following result shows that $X_{7}(T)$ and thus $X(T)$ as well can not be approximated in $p$-th mean sense with a polynomial error rate in terms of the number of evaluations of the driving Brownian motion $W$ as long as the number and the location of the evaluation nodes for $W$ are not chosen in a path-dependent way.

Theorem 1. There exists $c \in(0, \infty)$ such that for all $n \in \mathbb{N}$,

$$
\inf _{\substack{s_{1}, \ldots, s_{n} \in[0, T] \\ u: \mathbb{R}^{n} \rightarrow \mathbb{R} \text { measurable }}}\left(\mathbb{E}\left[\left|X_{7}(T)-u\left(W\left(s_{1}\right), \ldots, W\left(s_{n}\right)\right)\right|^{p}\right]\right)^{\frac{1}{p}} \geq c \cdot \frac{1}{\ln ^{\frac{2}{p}}(n+1)} .
$$

Our next result shows that a polynomial $p$-th mean error rate for approximation of $X(T)$ can be achieved if the number of the evaluation nodes for $W$ is adjusted to the current path of $W$.

For $n \in \mathbb{N}$ we use $\bar{W}_{n}:\left[0, \tau_{1}\right] \times \Omega \rightarrow \mathbb{R}$ to denote the piecewise linear interpolation of $W$ on $\left[0, \tau_{1}\right]$ at the nodes $t_{i}=i / n \cdot \tau_{1}, i=0, \ldots, n$, i.e.

$$
\bar{W}_{n}(t)=\frac{t-t_{i-1}}{\tau_{1} / n} \cdot W\left(t_{i}\right)+\frac{t_{i}-t}{\tau_{1} / n} \cdot W\left(t_{i-1}\right), \quad t \in\left[t_{i-1}, t_{i}\right],
$$

for $i \in\{1, \ldots, n\}$. We define approximations of the single components of $X(T)$ in the following way. Put

$$
\begin{aligned}
& \widehat{X}_{n, 1}(T)=T, \quad \widehat{X}_{n, 2}(T)=-\int_{0}^{\tau_{1}} f^{\prime}(t) \cdot \bar{W}_{n}(t) d t, \quad \widehat{X}_{n, 3}(T)=\widehat{X}_{n, 2}^{2}(T), \\
& \widehat{X}_{n, 4}(T)=0, \quad \widehat{X}_{n, 5}(T)=\exp \left(\frac{1}{4 p} \widehat{X}_{n, 2}^{2}\right), \quad \widehat{X}_{n, 6}(T)=0 .
\end{aligned}
$$

Next, let

$$
a_{\ell}=2 \sqrt{\ln \ell}
$$

for $\ell \in \mathbb{N}$ and put

$$
\widehat{X}_{n, 2}^{*}(T)=\sum_{\ell=1}^{\infty} \widehat{X}_{\ell n, 2}(T) \cdot 1_{\left[a_{\ell}, a_{\ell+1}\right)}\left(\left|\widehat{X}_{n, 2}(T)\right|\right) .
$$

Finally, define $G: \mathbb{R} \rightarrow \mathbb{R}$ by

$$
G(x)=\frac{\exp \left(\frac{1}{2 p} x^{2}\right)}{\left(1+x^{2}\right)^{\frac{1}{2 p}} \cdot \ln ^{\frac{2}{p}}\left(2+x^{2}\right)}, \quad x \in \mathbb{R}
$$

and put

$$
\widehat{X}_{n, 7}^{*}(T)=G\left(\widehat{X}_{n, 2}^{*}(T)\right)
$$

as well as

$$
\widehat{X}_{n}^{*}(T)=\left(\widehat{X}_{n, 1}(T), \ldots, \widehat{X}_{n, 6}(T), \widehat{X}_{n, 7}^{*}(T)\right) .
$$


APPROXIMATION OF SDES WITH SMOOTH COEFFIENTS WITH DERIVATIVES OF LINEAR GROWTH 9

Clearly, the random number of evaluations of $W$ used by the approximation $\widehat{X}_{n}^{*}(T)$ is given by

$$
\operatorname{cost}\left(\widehat{X}_{n}^{*}(T)\right)=n \sum_{\ell=1}^{\infty} \ell \cdot \mathbb{1}_{\left[a_{\ell}, a_{\ell+1}\right)}\left(\left|\widehat{X}_{n, 2}(T)\right|\right) .
$$

Theorem 2. There exists $c \in(0, \infty)$ such that for all $n \in \mathbb{N}$,

$$
\mathbb{E}\left[\operatorname{cost}\left(\widehat{X}_{n}^{*}(T)\right)\right] \leq c \cdot n \quad \text { and } \quad\left(\mathbb{E}\left[\left|X(T)-\widehat{X}_{n}^{*}(T)\right|^{p}\right]\right)^{\frac{1}{p}} \leq \frac{c}{n} .
$$

Finally, we show that for $q<p$ a polynomial $q$-th mean error rate for approximation of $X(T)$ can be achieved with a sequence of non-adaptive approximations. For $n \in \mathbb{N}$ put

$$
\widehat{X}_{n, 7}(T)=G\left(\widehat{X}_{n, 2}(T)\right)
$$

with $G$ given by (22) and define

$$
\widehat{X}_{n}(T)=\left(\widehat{X}_{n, i}(T)\right)_{i=1, \ldots, 7}
$$

Note that $\widehat{X}_{n}(T)=u_{n}\left(W\left(\tau_{1} / n\right), W\left(2 \tau_{1} / n\right), \ldots, W\left(\tau_{1}\right)\right)$ for some function $u_{n}: \mathbb{R}^{n} \rightarrow \mathbb{R}^{7}$.

Theorem 3. Let $q \in[0, p)$. Then there exists $c \in(0, \infty)$ such that for all $n \in \mathbb{N}$,

$$
\left(\mathbb{E}\left[\left|X(T)-\widehat{X}_{n}(T)\right|^{q}\right]\right)^{\frac{1}{q}} \leq \frac{c}{n} .
$$

\section{Proof of Theorem 1}

For the proof of Theorem 1 we employ the following lemma, which is a straightforward generalization of Lemma 4.1 in [20].

Lemma 3. Let $\left(\Omega_{1}, \mathcal{A}_{1}\right)$ and $\left(\Omega_{2}, \mathcal{A}_{2}\right)$ be measurable spaces and let $V_{1}: \Omega \rightarrow \Omega_{1}$ and $V_{2}, V_{2}^{\prime}, V_{2}^{\prime \prime}$ : $\Omega \rightarrow \Omega_{2}$ be random variables such that

$$
\mathbb{P}_{\left(V_{1}, V_{2}\right)}=\mathbb{P}_{\left(V_{1}, V_{2}^{\prime}\right)}=\mathbb{P}_{\left(V_{1}, V_{2}^{\prime \prime}\right)} .
$$

Then for all $q \in[1, \infty)$ and for all measurable mappings $\Phi: \Omega_{1} \times \Omega_{2} \rightarrow \mathbb{R}$ and $\varphi: \Omega_{1} \rightarrow \mathbb{R}$,

$$
\left(\mathbb{E}\left[\left|\Phi\left(V_{1}, V_{2}\right)-\varphi\left(V_{1}\right)\right|^{q}\right]\right)^{\frac{1}{q}} \geq \frac{1}{2}\left(\mathbb{E}\left[\left|\Phi\left(V_{1}, V_{2}^{\prime}\right)-\Phi\left(V_{1}, V_{2}^{\prime \prime}\right)\right|^{q}\right]\right)^{\frac{1}{q}} .
$$

We start with the proof of Theorem 1 Let $n \in \mathbb{N}$ and $s_{1}, \ldots, s_{n} \in[0, T]$. Clearly, there exist $0 \leq t_{0}<t_{1} \leq T$ such that

$$
\left[t_{0}, t_{1}\right] \subset\left[0, \tau_{1} / 2\right], \quad\left(t_{0}, t_{1}\right) \cap\left\{s_{1}, \ldots, s_{n}\right\}=\emptyset, \quad t_{1}-t_{0}=\frac{\tau_{1}}{2(n+1)} .
$$

Define processes $\bar{W}, B:\left[t_{0}, t_{1}\right] \times \Omega \rightarrow \mathbb{R}$ and $\widetilde{W}:\left(\left[0, t_{0}\right] \cup\left[t_{1}, T\right]\right) \times \Omega \rightarrow \mathbb{R}$ by

$$
\bar{W}(t)=\frac{\left(t-t_{0}\right)}{\left(t_{1}-t_{0}\right)} \cdot W\left(t_{1}\right)+\frac{\left(t_{1}-t\right)}{\left(t_{1}-t_{0}\right)} \cdot W\left(t_{0}\right), \quad B(t)=W(t)-\bar{W}(t)
$$


for $t \in\left[t_{0}, t_{1}\right]$ and by $\widetilde{W}(t)=W(t)$ for $t \in\left[0, t_{0}\right] \cup\left[t_{1}, T\right]$. Moreover, let

$$
\begin{aligned}
& Y_{1}=-\int_{0}^{t_{0}} f^{\prime}(s) \cdot W(s) d s-\int_{t_{0}}^{t_{1}} f^{\prime}(s) \cdot \bar{W}(s) d s-\int_{t_{1}}^{\tau_{1}} f^{\prime}(s) \cdot W(s) d s \\
& Y_{2}=-\int_{t_{0}}^{t_{1}} f^{\prime}(s) \cdot B(s) d s .
\end{aligned}
$$

By Itô's formula and (12) we have $\mathbb{P}$-a.s.

$$
Y_{1}+Y_{2}=\int_{0}^{\tau_{1}} f(s) d W(s)
$$

Hence, by (19), $\mathbb{P}$-a.s.

$$
X_{7}(T)=G\left(Y_{1}+Y_{2}\right)
$$

where $G: \mathbb{R} \rightarrow \mathbb{R}$ is given by (22).

Let $u: \mathbb{R}^{n} \rightarrow \mathbb{R}$ be a measurable mapping. Using (26) we obtain

$$
\mathbb{E}\left[\left|X_{7}(T)-u\left(W\left(s_{1}\right), \ldots, W\left(s_{n}\right)\right)\right|^{p}\right]=\mathbb{E}\left[\left|G\left(Y_{1}+Y_{2}\right)-u\left(W\left(s_{1}\right), \ldots, W\left(s_{n}\right)\right)\right|^{p}\right] .
$$

The first two statements in (24) imply that there exist measurable functions $\Phi_{1}, \varphi: C\left(\left[0, t_{0}\right] \cup\right.$ $\left.\left[t_{1}, T\right], \mathbb{R}\right) \rightarrow \mathbb{R}$ and $\Phi_{2}: C\left(\left[t_{0}, t_{1}\right], \mathbb{R}\right) \rightarrow \mathbb{R}$ such that

$$
Y_{1}=\Phi_{1}(\widetilde{W}), \quad Y_{2}=\Phi_{2}(B), \quad u\left(W\left(s_{1}\right), \ldots, W\left(s_{n}\right)\right)=\varphi(\widetilde{W}) .
$$

Moreover, $\widetilde{W}$ and $B$ are independent and $B$ has a symmetric distribution, which yields

$$
\mathbb{P}_{(\widetilde{W}, B)}=\mathbb{P}_{(\widetilde{W},-B)} \cdot
$$

We may thus apply Lemma 3 with $\Omega_{1}=C\left(\left[0, t_{0}\right] \cup\left[t_{1}, T\right], \mathbb{R}\right), \Omega_{2}=C\left(\left[t_{0}, t_{1}\right], \mathbb{R}\right), V_{1}=\widetilde{W}$, $V_{2}=V_{2}^{\prime}=B, V_{2}^{\prime \prime}=-B, \Phi(\tilde{w}, b)=G\left(\Phi_{1}(\tilde{w})+\Phi_{2}(b)\right)$ for $(\tilde{w}, b) \in \Omega_{1} \times \Omega_{2}$ and $\varphi$ as above, and observing the fact that $\Phi_{2}(-B)=-\Phi_{2}(B)$ we conclude that

$$
\mathbb{E}\left[\left|G\left(Y_{1}+Y_{2}\right)-u\left(W\left(s_{1}\right), \ldots, W\left(s_{n}\right)\right)\right|^{p}\right] \geq \frac{1}{2^{p}} \mathbb{E}\left[\left|G\left(Y_{1}+Y_{2}\right)-G\left(Y_{1}-Y_{2}\right)\right|^{p}\right] .
$$

For the analysis of the right hand side in (28) we first collect useful properties of the random variables $Y_{1}$ and $Y_{2}$ and the function $G$.

Clearly, $Y_{1}$ and $Y_{2}$ are centered normal Gaussian variables. Moreover, independence of $\widetilde{W}$ and $B$ implies independence of $Y_{1}$ and $Y_{2}$. Let $\sigma_{1}^{2}$ and $\sigma_{2}^{2}$ denote the variances of $Y_{1}$ and $Y_{2}$, respectively. Due to (25) and the fact that $\int_{0}^{\tau_{1}} f^{2}(t) d t=1$, see (14), we then have

$$
\sigma_{1}^{2}+\sigma_{2}^{2}=1 \text {. }
$$

Put

$$
\alpha=\inf _{t \in\left[0, \tau_{1} / 2\right]}\left|f^{\prime}(t)\right|^{2}, \quad \beta=\sup _{t \in\left[0, \tau_{1} / 2\right]}\left|f^{\prime}(t)\right|^{2}
$$

and note that $0<\alpha \leq \beta<\infty$, due to (13). Since

$$
\sigma_{2}^{2}=\int_{t_{0}}^{t_{1}} \int_{t_{0}}^{t_{1}} f^{\prime}(s) \cdot f^{\prime}(t) \cdot \frac{\left(t_{1}-\max (s, t)\right)\left(\min (s, t)-t_{0}\right)}{t_{1}-t_{0}} d s d t
$$


APPROXIMATION OF SDES WITH SMOOTH COEFFIENTS WITH DERIVATIVES OF LINEAR GROWTH 11 and $\left[t_{0}, t_{1}\right] \subset\left[0, \tau_{1} / 2\right]$ we conclude that

$$
\alpha \cdot \frac{\left(t_{1}-t_{0}\right)^{3}}{12} \leq \sigma_{2}^{2} \leq \beta \cdot \frac{\left(t_{1}-t_{0}\right)^{3}}{12} .
$$

Put

$$
n_{0}=\left\lceil\frac{\tau_{1}}{2} \cdot\left(\frac{\beta}{6}\right)^{1 / 3}-1\right\rceil .
$$

Using (24), (29) and (30) we obtain that if $n \geq n_{0}$ then

$$
\frac{\alpha \cdot \tau_{1}^{3}}{96(n+1)^{3}} \leq \sigma_{2}^{2} \leq 1 / 2 \leq \sigma_{1}^{2}, \quad \sigma_{1}^{-2}-1=\frac{\sigma_{2}^{2}}{\sigma_{1}^{2}} \leq \frac{\beta \cdot \tau_{1}^{3}}{48(n+1)^{3}} .
$$

Clearly, for all $x \geq 1$,

$$
G^{p}(x)=\frac{\exp \left(x^{2} / 2\right)}{\left(1+x^{2}\right)^{\frac{1}{2}} \cdot \ln ^{2}\left(2+x^{2}\right)} \geq \frac{\exp \left(x^{2} / 2\right)}{\sqrt{2} x \cdot \ln ^{2}\left(3 x^{2}\right)} .
$$

Moreover, $G$ is differentiable on $\mathbb{R}$ with

$$
G^{\prime}(x)=\frac{x}{p} \cdot G(x) \cdot\left(1-\frac{1}{\left(1+x^{2}\right)}-\frac{4}{\left(2+x^{2}\right) \cdot \ln \left(2+x^{2}\right)}\right) .
$$

Hence, for all $x \geq 3$,

$$
G^{\prime}(x) \geq \frac{x}{2 p} \cdot G(x)>0 .
$$

Clearly, we may assume that $n \geq \max \left(3, n_{0}\right)$. Let $y_{1} \in\left[n^{\frac{3}{2}}, 2 n^{\frac{3}{2}}\right]$ and $y_{2} \in\left[0, \sigma_{2}\right]$. Then $y_{1}+y_{2} \geq y_{1}-y_{2} \geq n^{\frac{3}{2}}-\sigma_{2} \geq 3^{\frac{3}{2}}-1>3$, due to (29). Hence, by (34),

$$
\left|G\left(y_{1}+y_{2}\right)-G\left(y_{1}-y_{2}\right)\right| \geq \int_{y_{1}}^{y_{1}+y_{2}} G^{\prime}(x) d x \geq \frac{1}{2 p} y_{1} \cdot y_{2} \cdot G\left(y_{1}\right),
$$

which jointly with (32) yields

$$
\left|G\left(y_{1}+y_{2}\right)-G\left(y_{1}-y_{2}\right)\right|^{p} \geq \frac{1}{(2 p)^{p}} y_{1}^{p-1} \cdot y_{2}^{p} \cdot \frac{\exp \left(y_{1}^{2} / 2\right)}{\sqrt{2} \cdot \ln ^{2}\left(3 y_{1}^{2}\right)} .
$$

Employing (29) and (31) we conclude that

$$
\begin{aligned}
\mathbb{E}\left[\mid G\left(Y_{1}+\right.\right. & \left.\left.Y_{2}\right)-\left.G\left(Y_{1}-Y_{2}\right)\right|^{p}\right] \\
& \geq \frac{1}{(2 p)^{p} 2^{\frac{3}{2}} \pi} \cdot \frac{1}{\sigma_{1} \sigma_{2}} \int_{n^{\frac{3}{2}}}^{2 n^{\frac{3}{2}}} \int_{0}^{\sigma_{2}} \frac{y_{1}^{p-1} y_{2}^{p}}{\ln ^{2}\left(3 y_{1}^{2}\right)} \cdot \exp \left(-\frac{y_{2}^{2}}{2 \sigma_{2}^{2}}-\frac{y_{1}^{2}}{2}\left(\sigma_{1}^{-2}-1\right)\right) d y_{2} d y_{1} \\
& \geq \frac{1}{(2 p)^{p} 2^{\frac{3}{2}} \pi} \cdot \frac{\sigma_{2}^{p}}{(p+1) \sqrt{e}} \int_{n^{\frac{3}{2}}}^{2 n^{\frac{3}{2}}} \frac{y_{1}^{p-1}}{\ln ^{2}\left(3 y_{1}^{2}\right)} \cdot \exp \left(-\frac{y_{1}^{2}}{2}\left(\sigma_{1}^{-2}-1\right)\right) d y_{1} \\
& \geq \frac{1}{(2 p)^{p} 2^{\frac{3}{2}} \pi} \cdot \frac{\sigma_{2}^{p}}{(p+1) \sqrt{e}} \cdot \frac{n^{\frac{3 p}{2}}}{\ln ^{2}\left(12 n^{3}\right)} \cdot \exp \left(-2 n^{3}\left(\sigma_{1}^{-2}-1\right)\right) \\
& \geq \frac{1}{(2 p)^{p}(p+1) 2^{\frac{3}{2}} \pi \sqrt{e}} \cdot\left(\frac{\tau_{1}^{3} \alpha}{96}\right)^{\frac{p}{2}} \cdot\left(\frac{n}{n+1}\right)^{\frac{3 p}{2}} \cdot \frac{\exp \left(-\frac{n^{3}}{24(n+1)^{3}} \cdot \beta \tau_{1}^{3}\right)}{\ln ^{2}\left(12 n^{3}\right)} \\
& \geq \frac{\left(\tau_{1}^{3} \alpha\right)^{\frac{p}{2}}}{2^{5 p+\frac{3}{2}} 3^{\frac{p}{2}} p^{p}(p+1) \pi \sqrt{e}} \cdot \exp \left(-\frac{\beta \tau_{1}^{3}}{24}\right) \cdot \frac{1}{\ln ^{2}\left(12 n^{3}\right)} \cdot
\end{aligned}
$$

Now combine (27), (28) and (35) to complete the proof of Theorem 1 , 


\section{Proof of Theorems 2 and 3}

As technical tools for the proof of Theorems 2 and 3 we employ the following two results for centered Gaussian random variables.

Lemma 4. For every $q \in[0, \infty)$ there exists $\kappa_{q} \in(0, \infty)$ such that for every random variable $Z \sim \mathcal{N}\left(0, \sigma^{2}\right)$ with $\sigma^{2} \in\left[0, \frac{1}{4}\right]$ and every $a \in[0, \infty)$

$$
\mathbb{E}\left[|Z|^{q} \cdot \exp \left(a \cdot|Z|+|Z|^{2}\right)\right] \leq \kappa_{q} \cdot \sigma^{q} \cdot\left(1+a^{q}\right) \cdot \exp \left(a^{2} \cdot \sigma^{2}\right) .
$$

Proof. Let $q, a \in[0, \infty)$ and let $Z \sim \mathcal{N}\left(0, \sigma^{2}\right)$ with $\sigma^{2} \in\left[0, \frac{1}{4}\right]$. Without loss of generality we may assume that $\sigma^{2}>0$. Let $V \sim \mathcal{N}(0,1)$. Then

$$
\begin{aligned}
\mathbb{E}\left[|Z|^{q} \cdot \exp \left(a \cdot|Z|+|Z|^{2}\right)\right] & =\frac{\sqrt{2}}{\sqrt{\pi} \sigma} \cdot \int_{0}^{\infty} x^{q} \cdot \exp \left(a x+x^{2}-\frac{x^{2}}{2 \sigma^{2}}\right) d x \\
& =\frac{\sqrt{2}}{\sqrt{\pi} \sigma} \cdot \exp \left(\frac{a^{2} \sigma^{2}}{2-4 \sigma^{2}}\right) \cdot \int_{0}^{\infty} x^{q} \cdot \exp \left(-\frac{1-2 \sigma^{2}}{2 \sigma^{2}} \cdot\left(x-\frac{a \sigma^{2}}{1-2 \sigma^{2}}\right)^{2}\right) d x \\
& \leq \exp \left(\frac{a^{2} \sigma^{2}}{2-4 \sigma^{2}}\right) \cdot \frac{2}{\sqrt{1-2 \sigma^{2}}} \cdot \mathbb{E}\left[\left|\frac{\sigma}{\sqrt{1-2 \sigma^{2}}} \cdot V+\frac{a \sigma^{2}}{1-2 \sigma^{2}}\right|^{q}\right] \\
& \leq \exp \left(\frac{a^{2} \sigma^{2}}{2-4 \sigma^{2}}\right) \cdot \frac{2^{q+1} \sigma^{q}}{\left(1-2 \sigma^{2}\right)^{\frac{q+1}{2}}} \cdot\left(\mathbb{E}\left[|V|^{q}\right]+a^{q} \frac{\sigma^{q}}{\left(1-2 \sigma^{2}\right)^{\frac{q}{2}}}\right) .
\end{aligned}
$$

Note that $\sigma^{2} \leq 1 / 4$ implies $1-2 \sigma^{2} \geq 1 / 2$ as well as $\sigma^{2} /\left(1-2 \sigma^{2}\right) \leq 1 / 2$, which finishes the proof of the lemma.

Lemma 5. Let $q \in[0, \infty)$ and $r \in\left[0, \frac{1}{2 q}\right)$ and let $H \in C^{1}(\mathbb{R}, \mathbb{R})$ with

$$
\sup _{x \in \mathbb{R}}\left|H^{\prime}(x)\right| \cdot \exp \left(-q \cdot x^{2}\right)<\infty .
$$

Then there exists $\kappa \in(0, \infty)$ such that for all independent random variables $V_{1} \sim \mathcal{N}\left(0, v_{1}^{2}\right)$, $V_{2} \sim \mathcal{N}\left(0, v_{2}^{2}\right)$ with $v_{1}^{2}+v_{1}^{2} \leq 1$

$$
\mathbb{E}\left[\left|H\left(V_{1}+V_{2}\right)-H\left(V_{1}\right)\right|^{r}\right] \leq \kappa \cdot v_{2}^{r} .
$$

Proof. Let $q \in[0, \infty)$ and $r \in\left[0, \frac{1}{2 q}\right)$, let $H \in C^{1}(\mathbb{R}, \mathbb{R})$ satisfy (36) and let $V_{1} \sim \mathcal{N}\left(0, v_{1}^{2}\right)$, $V_{2} \sim \mathcal{N}\left(0, v_{2}^{2}\right)$ be independent with $v_{1}^{2}+v_{1}^{2} \leq 1$. Let $U_{1}$ and $U_{2}$ be independent standard normal random variables. By the properties of $H$ there exists $c \in(0, \infty)$ such that for all $y, z \in \mathbb{R}$,

$$
|H(y+z)-H(y)| \leq \int_{\min (y, y+z)}^{\max (y, y+z)}\left|H^{\prime}(x)\right| d x \leq c \cdot|z| \cdot \exp \left(q \cdot(|y|+|z|)^{2}\right) .
$$

By the latter estimate, the Hölder inequality and the fact that $v_{1}^{2}+v_{2}^{2} \leq 1$ we get

$$
\begin{aligned}
\mathbb{E}\left[\left|H\left(V_{1}+V_{2}\right)-H\left(V_{1}\right)\right|^{r}\right] & \leq c^{r} \cdot v_{2}^{r} \cdot \mathbb{E}\left[\left|U_{2}\right|^{r} \cdot \exp \left(r \cdot q \cdot\left(v_{1} \cdot\left|U_{1}\right|+v_{2} \cdot\left|U_{2}\right|\right)^{2}\right)\right] \\
& \leq c^{r} \cdot v_{2}^{r} \cdot \mathbb{E}\left[\left|U_{2}\right|^{r} \cdot \exp \left(r \cdot q \cdot\left(U_{1}^{2}+U_{2}^{2}\right)\right)\right] \\
& =c^{r} \cdot v_{2}^{r} \cdot \mathbb{E}\left[\left|U_{2}\right|^{r} \cdot \exp \left(r \cdot q \cdot U_{2}^{2}\right)\right] \cdot \mathbb{E}\left[\exp \left(r \cdot q \cdot U_{1}^{2}\right)\right] \\
& \leq c^{r} \cdot v_{2}^{r} \cdot\left(\mathbb{E}\left[\left(1+\left|U_{2}\right|^{r}\right) \cdot \exp \left(r \cdot q \cdot U_{2}^{2}\right)\right]\right)^{2} \cdot
\end{aligned}
$$


Note that $r q<1 / 2$ and put $v=(1-2 r q)^{-1 / 2}$. Then

$$
\mathbb{E}\left[\left(1+\left|U_{2}\right|^{r}\right) \cdot \exp \left(r \cdot q \cdot U_{2}^{2}\right)\right]=\int_{\mathbb{R}} \frac{\left(1+|x|^{r}\right)}{\sqrt{2 \pi}} \cdot \exp \left(\frac{-x^{2}}{2 v^{2}}\right) d x=v \cdot\left(1+v^{r} \cdot \mathbb{E}\left[\left|U_{1}\right|^{r}\right]\right),
$$

which completes the proof of the lemma.

In the sequel we use the following notation. For $n \in \mathbb{N}$ we define $B_{n}:\left[0, \tau_{1}\right] \times \Omega \rightarrow \mathbb{R}$ by

$$
B_{n}(t)=W(t)-\bar{W}_{n}(t), \quad t \in\left[0, \tau_{1}\right],
$$

and we put

$$
Y_{n}=-\int_{0}^{\tau_{1}} f^{\prime}(t) B_{n}(t) d t, \quad Z_{n}=\widehat{X}_{n, 2}(T)
$$

as well as

$$
\sigma_{n}^{2}=\operatorname{Var}\left(Y_{n}\right), \quad \nu_{n}^{2}=\operatorname{Var}\left(Z_{n}\right)
$$

By Itô's formula and (12) we have $\mathbb{P}$-a.s.

$$
X_{2}(T)=Z_{n}+Y_{n}
$$

Let $n \in \mathbb{N}$ and $\ell \in \mathbb{N}$. Then it is easy to check that

$$
Z_{n}, Z_{\ell n}-Z_{n}, Y_{\ell n} \text { are independent, centered, Gaussian random variables. }
$$

Moreover, using (39) and Lemma 2 we get

$$
\operatorname{Var}\left(Y_{\ell n}\right)+\operatorname{Var}\left(Z_{\ell n}\right)=\sigma_{\ell n}^{2}+\nu_{\ell n}^{2}=1, \quad \operatorname{Var}\left(Z_{\ell n}-Z_{n}\right)=\nu_{\ell n}^{2}-\nu_{n}^{2},
$$

and, proceeding as in the proof of (30), it is easy to see that

$$
\sigma_{\ell n}^{2} \leq \gamma \frac{\tau_{1}^{3}}{12 \ell^{2} n^{2}}
$$

where $\gamma=\sup _{t \in\left[0, \tau_{1}\right]}\left|f^{\prime}(t)\right|^{2}$.

We are ready to establish a $p$-th mean error estimate for the approximation $\widehat{X}_{n, 7}^{*}(T)$.

Lemma 6. There exists $c \in(0, \infty)$ such that for all $n \in \mathbb{N}$,

$$
\mathbb{E}\left[\left|X_{7}(T)-\widehat{X}_{n, 7}^{*}(T)\right|^{p}\right] \leq \frac{c}{n^{p}} .
$$

Proof. Let $n \in \mathbb{N}$. Using (39) we obtain

$$
\begin{aligned}
\mathbb{E}\left[\left|X_{7}(T)-\widehat{X}_{n, 7}^{*}(T)\right|^{p}\right] & =\mathbb{E}\left[\left|G\left(X_{2}(T)\right)-G\left(\widehat{X}_{n, 2}^{*}(T)\right)\right|^{p}\right] \\
& =\sum_{\ell=1}^{\infty} \mathbb{E}\left[\left|G\left(Z_{\ell n}+Y_{\ell n}\right)-G\left(Z_{\ell n}\right)\right|^{p} \cdot 1_{\left[a_{\ell}, a_{\ell+1}\right)}\left(\left|Z_{n}\right|\right)\right] .
\end{aligned}
$$

It follows from (33) that there exists $c_{1} \in(0, \infty)$ such that

$$
\left|G^{\prime}(x)\right| \leq c_{1} \cdot|x| \cdot \exp \left(\frac{x^{2}}{2 p}\right)
$$

for all $x \in \mathbb{R}$. Hence, for all $y, z \in \mathbb{R}$,

$$
|G(z+y)-G(z)| \leq \int_{\min (z, z+y)}^{\max (z, z+y)}\left|G^{\prime}(x)\right| d x \leq c_{1} \cdot|y| \cdot(|z|+|y|) \cdot \exp \left(\frac{1}{2 p}(|z|+|y|)^{2}\right),
$$


which jointly with (43) implies

$$
\begin{aligned}
& \mathbb{E}\left[\left|X_{7}(T)-\widehat{X}_{n, 7}^{*}(T)\right|^{p}\right] \\
& \quad \leq c_{1}^{p} \cdot \sum_{\ell=1}^{\infty} \mathbb{E}\left[\left|Y_{\ell n}\right|^{p} \cdot\left(\left|Z_{\ell n}\right|+\left|Y_{\ell n}\right|\right)^{p} \cdot \exp \left(\frac{1}{2}\left(\left|Z_{\ell n}\right|+\left|Y_{\ell n}\right|\right)^{2}\right) \cdot 1_{\left[a_{\ell}, a_{\ell+1}\right)}\left(\left|Z_{n}\right|\right)\right] .
\end{aligned}
$$

Note that for all $\ell \in \mathbb{N}$,

$$
\begin{aligned}
& \exp \left(\frac{1}{2}\left(\left|Z_{\ell n}\right|+\left|Y_{\ell n}\right|\right)^{2}\right) \cdot 1_{\left[a_{\ell}, a_{\ell+1}\right)}\left(\left|Z_{n}\right|\right) \\
& \quad \leq \exp \left(\frac{1}{2}\left|Z_{n}\right|^{2}+a_{\ell+1} \cdot\left(\left|Z_{\ell n}-Z_{n}\right|+\left|Y_{\ell n}\right|\right)+\left|Z_{\ell n}-Z_{n}\right|^{2}+\left|Y_{\ell n}\right|^{2}\right) \cdot 1_{\left[a_{\ell}, a_{\ell+1}\right)}\left(\left|Z_{n}\right|\right)
\end{aligned}
$$

and

$$
\left(\left|Z_{\ell n}\right|+\left|Y_{\ell n}\right|\right)^{p} \leq 3^{p} \cdot\left(1+\left|Z_{n}\right|^{p}\right) \cdot\left(1+\left|Z_{\ell n}-Z_{n}\right|^{p}\right) \cdot\left(1+\left|Y_{\ell n}\right|^{p}\right) .
$$

Hence,

$$
\mathbb{E}\left[\left|X_{7}(T)-\widehat{X}_{n, 7}^{*}(T)\right|^{p}\right] \leq\left(3 c_{1}\right)^{p} \cdot \sum_{\ell=1}^{\infty} \mathbb{E}\left[A_{\ell, n} \cdot B_{\ell, n} \cdot C_{\ell, n}\right],
$$

where

$$
\begin{aligned}
& A_{\ell, n}=\left(1+\left|Z_{n}\right|^{p}\right) \cdot \exp \left(\frac{1}{2}\left|Z_{n}\right|^{2}\right) \cdot 1_{\left[a_{\ell}, a_{\ell+1}\right)}\left(\left|Z_{n}\right|\right), \\
& B_{\ell, n}=\left(1+\left|Z_{\ell n}-Z_{n}\right|^{p}\right) \cdot \exp \left(a_{\ell+1} \cdot\left|Z_{\ell n}-Z_{n}\right|+\left|Z_{\ell n}-Z_{n}\right|^{2}\right), \\
& C_{\ell, n}=\left|Y_{\ell n}\right|^{p} \cdot\left(1+\left|Y_{\ell n}\right|^{p}\right) \cdot \exp \left(a_{\ell+1} \cdot\left|Y_{\ell n}\right|+\left|Y_{\ell n}\right|^{2}\right)
\end{aligned}
$$

for $\ell \in \mathbb{N}$. Observe that (40) implies that for all $\ell \in \mathbb{N}$,

$$
\mathbb{E}\left[A_{\ell, n} \cdot B_{\ell, n} \cdot C_{\ell, n}\right]=\mathbb{E}\left[A_{\ell, n}\right] \cdot \mathbb{E}\left[B_{\ell, n}\right] \cdot \mathbb{E}\left[C_{\ell, n}\right] .
$$

Next, put

$$
n_{1}=\left\lceil\sqrt{\gamma \tau_{1}^{3}}\right\rceil .
$$

Using (41) and (42) we see that for all $n \geq n_{1}$ and $\ell \in \mathbb{N}$,

$$
\nu_{\ell n}^{2}-\nu_{n}^{2}=\sigma_{n}^{2}-\sigma_{\ell n}^{2} \leq \sigma_{n}^{2} \leq \frac{\gamma \tau_{1}^{3}}{12 n^{2}} \leq \frac{1}{12} .
$$

Using (47), (42) and Lemma 4 we thus obtain that there exist $\kappa_{0}, \kappa_{p}, \kappa_{2 p}, c_{2}, c_{3} \in(0, \infty)$ such that for all $n \geq n_{1}$ and $\ell \in \mathbb{N}$,

$$
\mathbb{E}\left[B_{\ell, n}\right] \leq\left(2 \kappa_{0}+\kappa_{p} \cdot\left(\nu_{\ell n}^{2}-\nu_{n}^{2}\right)^{\frac{p}{2}} \cdot\left(1+a_{\ell+1}^{p}\right)\right) \cdot \exp \left(a_{\ell+1}^{2} \cdot\left(\nu_{\ell n}^{2}-\nu_{n}^{2}\right)\right) \leq c_{3} \cdot \ln ^{\frac{p}{2}}(\ell+1)
$$

and

$$
\mathbb{E}\left[C_{\ell, n}\right] \leq\left(\kappa_{p} \cdot \sigma_{\ell n}^{p} \cdot\left(1+a_{\ell+1}^{p}\right)+\kappa_{2 p} \cdot \sigma_{\ell n}^{2 p} \cdot\left(1+a_{\ell+1}^{2 p}\right)\right) \cdot \exp \left(a_{\ell+1}^{2} \cdot \sigma_{\ell n}^{2}\right) \leq \frac{c_{2}}{(\ell n)^{p}} \cdot \ln ^{p}(\ell+1) \cdot \ell^{\frac{1}{3}} .
$$

Furthermore, (41) and (47) jointly imply that $11 / 12 \leq \nu_{n}^{2} \leq 1$ for all $n \geq n_{1}$, and therefore there exists $c_{4} \in(0, \infty)$ such that for all $n \geq n_{1}$ and $\ell \in \mathbb{N}$,

$$
\begin{aligned}
\mathbb{E}\left[A_{\ell, n}\right] & =\frac{2}{\sqrt{2 \pi}} \int_{a_{\ell} / \nu_{n}}^{a_{\ell+1} / \nu_{n}}\left(1+\left(\nu_{n} \cdot x\right)^{p}\right) \cdot \exp \left(-\frac{x^{2}}{2} \cdot\left(1-\nu_{n}^{2}\right)\right) d x \leq\left(1+a_{\ell+1}^{p}\right) \cdot \frac{a_{\ell+1}-a_{\ell}}{\nu_{n}} \\
& \leq \sqrt{\frac{12}{11}}\left(1+2^{p} \ln ^{\frac{p}{2}}(\ell+1)\right) \cdot \frac{2}{\ell \cdot \ln ^{\frac{1}{2}}(\ell+1)} \leq c_{4} \cdot \ln ^{\frac{p-1}{2}}(\ell+1) \cdot \frac{1}{\ell} .
\end{aligned}
$$


Hence, there exists $c_{5} \in(0, \infty)$ such that for all $n \geq n_{1}$ and $\ell \in \mathbb{N}$,

$$
\mathbb{E}\left[A_{\ell, n}\right] \cdot \mathbb{E}\left[B_{\ell, n}\right] \cdot \mathbb{E}\left[C_{\ell, n}\right] \leq c_{5} \cdot \frac{1}{n^{p}} \cdot \frac{\ln ^{2 p-\frac{1}{2}}(\ell+1)}{\ell^{p+\frac{2}{3}}} .
$$

Combining (45), (46) and (48) we conclude that there exists $c_{6} \in(0, \infty)$ such that for all $n \geq n_{1}$,

$$
\mathbb{E}\left[\left|X_{7}(T)-\widehat{X}_{n, 7}^{*}(T)\right|^{p}\right] \leq\left(3 c_{1}\right)^{p} \cdot c_{5} \cdot \frac{1}{n^{p}} \sum_{\ell=1}^{\infty} \frac{\ln ^{2 p-\frac{1}{2}}(\ell+1)}{\ell^{p+\frac{2}{3}}} \leq c_{6} \cdot \frac{1}{n^{p}} .
$$

In view of Lemma 2 it remains to prove that for all $n<n_{1}$,

$$
\mathbb{E}\left[\left|G\left(\widehat{X}_{n, 2}^{*}(T)\right)\right|^{p}\right]<\infty .
$$

To this end we define $\rho: \mathbb{R} \rightarrow[3, \infty)$ by $\rho(x)=\max (|x|, 3)$. Clearly, $\rho$ is convex. Moreover, by (33) and (34) we obtain that $G$ as well as $G^{\prime}$ are increasing on $[3, \infty)$. In particular, $G$ is convex on $[3, \infty)$. Using the monotonicity and convexity of $G$ as well as the convexity of $\rho$ we conclude that $G \circ \rho$ is convex.

For $n, \ell \in \mathbb{N}$ put $\mathcal{F}_{\ell n}=\sigma\left(\left\{W\left(i \tau_{1} /(\ell n)\right): i=1, \ldots, \ell n\right\}\right)$ and note that $Z_{\ell n}=\mathbb{E}\left[X_{2}(T) \mid \mathcal{F}_{\ell n}\right]$. Using the estimate $G(x) \leq(\ln (2))^{-2 / p} \exp \left(x^{2} / 2 p\right)$ and the Jentzen inequality we therefore obtain that for all $n, \ell \in \mathbb{N}$

$$
\begin{aligned}
& \left|G\left(Z_{\ell n}\right)\right|^{p} \cdot \mathbb{1}_{\left[a_{\ell}, a_{\ell+1}\right)}\left(\left|Z_{n}\right|\right) \\
& \quad \leq\left(\left|G\left(\rho\left(Z_{\ell n}\right)\right)\right|^{p}+(\ln (2))^{-2} \exp (9 / 2)\right) \cdot \mathbb{1}_{\left[a_{\ell}, a_{\ell+1}\right)}\left(\left|Z_{n}\right|\right) \\
& \quad \leq\left(\mathbb{E}\left[\left|G\left(\rho\left(X_{2}(T)\right)\right)\right|^{p} \mid \mathcal{F}_{\ell n}\right]+(\ln (2))^{-2} \exp (9 / 2)\right) \cdot \mathbb{1}_{\left[a_{\ell}, a_{\ell+1}\right)}\left(\left|Z_{n}\right|\right) \\
& \quad=\mathbb{E}\left[\left|G\left(\rho\left(X_{2}(T)\right)\right)\right|^{p} \mathbb{1}_{\left[a_{\ell}, a_{\ell+1}\right)}\left(\left|Z_{n}\right|\right) \mid \mathcal{F}_{\ell n}\right]+(\ln (2))^{-2} \exp (9 / 2) \cdot \mathbb{1}_{\left[a_{\ell}, a_{\ell+1}\right)}\left(\left|Z_{n}\right|\right) .
\end{aligned}
$$

Hence there exists $c_{7} \in(0, \infty)$ such that for all $n \in \mathbb{N}$,

$$
\left|G\left(\widehat{X}_{n, 2}^{*}(T)\right)\right|^{p} \leq c_{7} \cdot\left(\sum_{\ell=1}^{\infty} \mathbb{E}\left[\left|G\left(\rho\left(X_{2}(T)\right)\right)\right|^{p} \mathbb{1}_{\left[a_{\ell}, a_{\ell+1}\right)}\left(\left|Z_{n}\right|\right) \mid \mathcal{F}_{\ell n}\right]+1\right),
$$

which implies

$$
\mathbb{E}\left[\left|G\left(\widehat{X}_{n, 2}^{*}(T)\right)\right|^{p}\right] \leq c_{7} \cdot\left(\mathbb{E}\left[\left|G\left(\rho\left(X_{2}(T)\right)\right)\right|^{p}\right]+1\right) .
$$

Finally, note that $G\left(\rho\left(X_{2}(T)\right)\right) \leq G\left(X_{2}(T)\right)+(\ln (2))^{-2 / p} \exp (9 / 2 p)$ and apply Lemma 2 to complete the proof of (50).

Next, we provide error estimates for the approximations $\widehat{X}_{n, 2}(T), \widehat{X}_{n, 3}(T), \widehat{X}_{n, 5}(T)$ and $\widehat{X}_{n, 7}(T)$.

Lemma 7. Let $r_{2}, r_{3} \in(0, \infty), r_{5} \in(0,2 p)$ and $r_{7} \in(0, p)$. Then there exists $c \in(0, \infty)$ such that for every $i \in\{2,3,5,7\}$ and every $n \in \mathbb{N}$,

$$
\mathbb{E}\left[\left|X_{i}(T)-\widehat{X}_{n, i}(T)\right|^{r_{i}}\right] \leq \frac{c}{n^{r_{i}}} .
$$

Proof. Let $r_{2}, r_{3} \in(0, \infty), r_{5} \in(0,2 p)$ and $r_{7} \in(0, p)$. Let $n \in \mathbb{N}$. In all of the four cases we apply Lemma 5 with $V_{1}=\widehat{X}_{n, 2}(T)=Z_{n}$ and $V_{2}=Y_{n}$, see (39). Thus $\operatorname{Var}\left(V_{1}\right)+\operatorname{Var}\left(V_{2}\right)=1$, 
and according to (42) we have $\operatorname{Var}\left(V_{2}\right) \leq\left(\gamma \tau_{1}^{3}\right) /\left(12 n^{2}\right)$. Moreover, for $i \in\{2,3,5,7\}$ we use the function $H=H_{i}$ in Lemma 5 , where $H_{2}, H_{3}, H_{5}, H_{7}: \mathbb{R} \rightarrow \mathbb{R}$ are given by

$$
H_{2}(x)=x, \quad H_{3}(x)=x^{2}, \quad H_{5}(x)=\exp \left(\frac{1}{4 p} x^{2}\right), \quad H_{7}(x)=G(x) .
$$

Let

$$
q_{2}=0, \quad q_{3} \in\left(0,1 /\left(2 r_{3}\right)\right), \quad q_{5} \in\left(1 /(4 p), 1 /\left(2 r_{5}\right)\right), \quad q_{7} \in\left(1 /(2 p), 1 /\left(2 r_{7}\right)\right) .
$$

Employing (44) in the case $i=7$ we then see that there exists $c \in(0, \infty)$ such that for every $i \in\{2,3,5,7\}$ and every $x \in \mathbb{R}$,

$$
\left|H_{i}^{\prime}(x)\right| \leq c \cdot \exp \left(q_{i} \cdot x^{2}\right)
$$

which completes the proof.

Clearly, Lemmas 6 and 7 jointly yield the error estimates in Theorems 2 and 3 ,

It remains to establish the cost estimate in Theorem 2.

Let $n \in \mathbb{N}$. Clearly, if $\nu_{n}=0$ then $\widehat{X}_{n, 2}(T)=Z_{n}=0$ a.s. and we have $\operatorname{cost}\left(\widehat{X}_{n}^{*}(T)\right)=n$ a.s. Next, assume $\nu_{n}^{2}>0$. Using (40) and the fact that $\nu_{n}^{2} \leq 1$ we get for every $l \in \mathbb{N}$,

$$
\begin{aligned}
\mathbb{P}\left(\left\{\left|Z_{n}\right| \in\left[a_{\ell}, a_{\ell+1}\right)\right\}\right) & =\frac{2}{\sqrt{2 \pi} \nu_{n}} \int_{a_{\ell}}^{a_{\ell+1}} \exp \left(-\frac{x^{2}}{2 \nu_{n}^{2}}\right) d x \leq \frac{2}{\sqrt{2 \pi} \nu_{n}} \cdot \exp \left(-\frac{a_{l}^{2}}{2 \nu_{n}^{2}}\right) \cdot\left(a_{\ell+1}-a_{\ell}\right) \\
& \leq \frac{1}{\nu_{n}} \cdot \frac{\sqrt{2}}{\sqrt{\pi}} \cdot \exp (-2 \ln (\ell)) \cdot \frac{2}{\sqrt{\ln (2) \cdot \ell}} \leq \frac{1}{\nu_{n}} \cdot \frac{2^{3 / 2}}{\sqrt{\pi \ln (2)}} \cdot \frac{1}{\ell^{3}} .
\end{aligned}
$$

Hence

$$
\mathbb{E}\left[\operatorname{cost}\left(\widehat{X}_{n}^{*}(T)\right)\right]=\sum_{\ell=1}^{\infty} \ell \cdot n \cdot \mathbb{P}\left(\left\{\left|Z_{n}\right| \in\left[a_{\ell}, a_{\ell+1}\right)\right\}\right) \leq \frac{n}{\nu_{n}} \cdot \frac{\sqrt{2}}{\sqrt{\pi \ln (2)}} \cdot \sum_{\ell=1}^{\infty} \frac{1}{\ell^{2}} .
$$

Finally note that $\lim _{n \rightarrow \infty} \nu_{n}^{2}=1$, due to (41) and (42), and therefore $\inf _{n: \nu_{n}>0} \nu_{n}>0$, which completes the proof of the cost estimate and finishes the proof of Theorems 2 and 3 ,

\section{Discussion}

The key contribution of this paper is to show that even then when an autonomous SDE on $[0, T]$ has smooth coefficients with first order derivatives of at most linear growth and its solution $X$ satisfies $\mathbb{E}\left[\sup _{t \in[0, T]}|X(t)|^{p}\right]<\infty$, where $p \in[1, \infty)$, it may happen that $X(T)$ can not be approximated on the basis of finitely many observations of the driving Brownian motion at fixed times in $[0, T]$ with a polynomial $p$-th mean error rate, see Theorem 1, This result naturally leads to a number of questions related to possible extensions or tightenings with respect to the class of approximations, the speed of convergence, the moment conditions on the solution and the polynomial growth conditions on the first order derivatives of the coefficients.

Does there exist an SDE of the above type such that a sub-polynomial rate of convergence holds for any adaptive approximation as well? For the SDE considered in the present paper, there is an adaptive method, which achieves a polynomial error rate, see Theorem 2 ,

Does there exist an SDE of the above type such that the smallest possible $p$-th mean error that can be achieved by any non-adaptive method based on $n$ evaluations of the driving Brownian motion or even by any adaptive method based on $n$ evaluations of the driving Brownian motion on average converges to zero slower than a given arbitrarily slow decay in terms of $n$ ? A negative 
result of this type is true for the class of SDEs with bounded smooth coefficients, see (2) and [20, 8, 36].

The first order derivatives of the coefficients of the pathological SDE (10) constructed in the present paper are of at most linear growth. Can a sub-polynomial rate of convergence of the smallest possible $p$-th mean error also occur when the first order derivatives of the coefficients are of at most polynomial growth with an exponent $\alpha \in(0,1)$ ?

Finally it is open, whether a sub-polynomial rate of convergence of the smallest possible $p$ th mean error can also occur when the solution $X$ has finite moments of some order $q>p$ or even satisfies $\mathbb{E}\left[\sup _{t \in[0, T]}|X(t)|^{q}\right]<\infty$ for all $q \geq 1$. The pathological SDE (10) satisfies $\mathbb{E}\left[\sup _{t \in[0, T]}|X(t)|^{q}\right]<\infty$ only for $q \leq p$, see Lemma 2 ,

\section{REFERENCES}

[1] Alfonsi, A. Strong order one convergence of a drift implicit Euler scheme: Application to the CIR process. Statist. Probab. Lett. 83, 2 (2013), 602-607.

[2] Berkaoui, A., Bossy, M., And Diop, A. Euler scheme for SDEs with non-Lipschitz diffusion coefficient: strong convergence. ESAIM Probab. Stat. 12 (2008), 1-11 (electronic).

[3] Beyn, W.-J., IsaAk, E., And Kruse, R. Stochastic C-stability and B-consistency of explicit and implicit Euler-type schemes. J. Sci. Comput. 67 (2015), 1-33.

[4] Beyn, W.-J., IsaAk, E., And Kruse, R. Stochastic C-stability and B-consistency of explicit and implicit Milstein-type schemes. J. Sci. Comput. 70 (2017), 1042-1077.

[5] Dereich, S., Neuenkirch, A., And Szpruch, L. An Euler-type method for the strong approximation of the Cox-Ingersoll-Ross process. Proc. R. Soc. Lond. Ser. A Math. Phys. Eng. Sci. 468, 2140 (2012), 1105-1115.

[6] Fang, W., And Giles, M. Adaptive Euler-Maruyama method for SDEs with non-globally Lipschitz drift: Part I, Finite Time Interval. arXiv:1609.08101 (2016), 31 pages.

[7] Gaines, J. G., And Lyons, T. J. Variable step size control in the numerical solution of stochastic differential equations. SIAM J. Appl. Math. 57, 5 (1997), 1455-1484.

[8] Gerencsér, M., Jentzen, A., And Salimova, D. On stochastic differential equations with arbitrarily slow convergence rates for strong approximation in two space dimensions. To appear in Proc. R. Soc. Lond. Ser. A Math. Phys. Eng. Sci.; arXiv:1702.03229 (2017), 31 pages.

[9] Gyöngy, I., And RÁsonyi, M. A note on Euler approximations for SDEs with Hölder continuous diffusion coefficients. Stochastic Process. Appl. 121, 10 (2011), 2189-2200.

[10] Hairer, M., Hutzenthaler, M., And Jentzen, A. Loss of regularity for Kolmogorov equations. Ann. Probab. 43, 2 (2015), 468-527.

[11] Hefter, M., and Herzwurm, A. Optimal strong approximation of the one-dimensional squared Bessel process. To appear in Commun. Math. Sci.; arXiv:1601.01455 (2016).

[12] Hefter, M., And Herzwurm, A. Strong convergence rates for Cox-Ingersoll-Ross processes - full parameter range. arXiv:1608.00410 (2016), 18 pages.

[13] Higham, D. J., Mao, X., And Stuart, A. M. Strong convergence of Euler-type methods for nonlinear stochastic differential equations. SIAM J. Numer. Anal. 40, 3 (2002), 1041-1063 (electronic).

[14] Hoel, H., von Schwerin, E., Szepessy, A., And Tempone, R. Adaptive multilevel Monte Carlo simulation. In Numerical analysis of multiscale computations, vol. 82 of Lect. Notes Comput. Sci. Eng. Springer, Heidelberg, 2012, pp. 217-234.

[15] Hoel, H., von Schwerin, E., Szepessy, A., And Tempone, R. Implementation and analysis of an adaptive multilevel Monte Carlo algorithm. Monte Carlo Methods Appl. 20, 1 (2014), 1-41.

[16] Hu, Y. Semi-implicit Euler-Maruyama scheme for stiff stochastic equations. In Stochastic analysis and related topics, V (Silivri, 1994), vol. 38 of Progr. Probab. Birkhäuser Boston, Boston, MA, 1996, pp. 183-202. 
[17] Hutzenthaler, M., And Jentzen, A. On a perturbation theory and on strong convergence rates for stochastic ordinary and partial differential equations with non-globally monotone coefficients. arXiv:1401.0295 (2014), 41 pages.

[18] Hutzenthaler, M., Jentzen, A., And Kloeden, P. E. Strong convergence of an explicit numerical method for SDEs with non-globally Lipschitz continuous coefficients. Ann. Appl. Probab. 22, 4 (2012), 1611-1641.

[19] Hutzenthaler, M., Jentzen, A., And Noll, M. Strong convergence rates and temporal regularity for Cox-Ingersoll-Ross processes and Bessel processes with accessible boundaries. arXiv: (2014), 32 pages.

[20] Jentzen, A., Müller-Gronbach, T., And Yaroslavtseva, L. On stochastic differential equations with arbitrary slow convergence rates for strong approximation. Commun. Math. Sci. 14, 7 (2016), 1477-1500.

[21] Kumar, C., And Sabanis, S. On Milstein approximations with varying coefficients: the case of super-linear diffusion coefficients. arXiv:1601.02695 (2016), 31 pages.

[22] Lamba, H., Mattingly, J. C., And Stuart, A. M. An adaptive Euler-Maruyama scheme for SDEs: convergence and stability. IMA J. Numer. Anal. 27, 3 (2007), 479-506.

[23] Leobacher, G., And SzÖlgyenyi, M. A strong order 1/2 method for multidimensional SDEs with discontinuous drift. To appear in Ann. Appl. Probab.; arXiv:1512.02807 (2015).

[24] Leobacher, G., And SzÖlgyenyi, M. A numerical method for SDEs with discontinuous drift. BIT 56, 1 (2016), 151-162.

[25] MaO, X., And SzPruch, L. Strong convergence rates for backward Euler-Maruyama method for non-linear dissipative-type stochastic differential equations with super-linear diffusion coefficients. Stochastics 85, 1 (2013), 144-171.

[26] Maruyama, G. Continuous Markov processes and stochastic equations. Rend. Circ. Mat. Palermo (2) 4 (1955), 48-90.

[27] Moon, K.-S., Szepessy, A., Tempone, R., and Zouraris, G. E. Convergence rates for adaptive weak approximation of stochastic differential equations. Stoch. Anal. Appl. 23, 3 (2005), 511-558.

[28] Müller-Gronbach, T. Strong approximation of systems of stochastic differential equations. Habilitation thesis, TU Darmstadt (2002), iv+161.

[29] Müller-Gronbach, T. Optimal pointwise approximation of SDEs based on Brownian motion at discrete points. Ann. Appl. Probab. 14, 4 (2004), 1605-1642.

[30] Neuenkirch, A., And SzPruch, L. First order strong approximations of scalar SDEs defined in a domain. Numerische Mathematik 128 (2014), 103-136.

[31] NGo, H.-L., And Taguchi, D. Strong rate of convergence for the Euler-Maruyama approximation of stochastic differential equations with irregular coefficients. Math. Comp. 85, 300 (2016), 1793-1819.

[32] Römisch, W., ANd Winkler, R. Stepsize control for mean-square numerical methods for stochastic differential equations with small noise. SIAM J. Sci. Comput. 28, 2 (2006), 604-625 (electronic).

[33] Sabanis, S. A note on tamed Euler approximations. Electron. Commun. Probab. 18 (2013), 1-10.

[34] Tretyakov, M., And Zhang, Z. A fundamental mean-square convergence theorem for SDEs with locally Lipschitz coefficients and its applications. SIAM J. Numer. Anal. 51, 6 (2013), 3135-3162.

[35] Wang, X., And Gan, S. The tamed Milstein method for commutative stochastic differential equations with non-globally Lipschitz continuous coefficients. J. Difference Equ. Appl. 19, 3 (2013), 466-490.

[36] Yaroslavtseva, L. On non-polynomial lower error bounds for adaptive strong approximation of sdes. To appear in J. Complexity; arXiv:1609.08073 (2016).

Fakultät für Informatik und Mathematik, Universität Passau, Innstrasse 33, 94032 Passau, GerMANY

E-mail address: larisa.yaroslavtseva@uni-passau.de, thomas.mueller-gronbach@uni-passau.de 